\title{
Développement mental des hypothyroïdiens congénitaux : le succès du dépistage
}

\author{
Jacqueline Glorieux \\ Réseau de médecine génétique du Québec
}

Remerciements

Ce travail a été réalisé grâce à la subvention refue du Fonds de la Recherche en Santé du Québec et de IUniversité de Montréal. L'auteur remercie Manon Desjardins et Jean Morissette pour leur contribution à cet article et Sylvie Tassé pour Tavoir dactylographié.

\section{Summary}

The Quebec Network for Genetic Medicine has followed the development of about Ioo hypothyroid children detected at one month of age and evaluated at I, I.5, 3, 5 and 7 years with the Griffiths mental development scales and the WISCR. Results to date show that children are developing normally but have lower scores than matched controls and siblings paired for age. The measure of knee bone surface, made before therapy was initiated, seems to be the best index of eventual mental outcome.

\author{
ADRESSE \\ J. Glorieux : Centre de Recherche Pédiatrique, \\ Hôpital Sainte-Justine, 3175, Chemin Côte- \\ Sainte-Catherine, Montréal, Québec, Canada, \\ $\mathrm{H}_{3} \mathrm{~T}_{1} \mathrm{C}_{5}$.
}

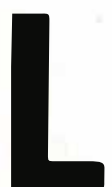

a justification des programmes de dépistage des maladies métaboliques repose sur l'amélioration du pronostic physique et mental des enfants traités précocément. Ce fut le cas pour les patients phénylcétonuriques et c'est l'espoir sur lequel repose le dépistage systématique de l'hypothyroïdie congénitale (HC). Ce déficit total ou partiel est compensé par une thérapie simple et efficace, qui demeure indispensable tout au long de la vie du patient; la prise régulière d'un comprimé par jour suffit à rétablir la croissance normale. Il convient d'augmenter régulièrement la dose de thyroxine au fur et à mesure de la croissance de l'enfant. Le nombre imposant de cas détectés chaque année dans le monde indique l'importance du problème [I]. Notre équipe a effectué pendant une période de presque to ans le suivi longitudinal du développement psychomoteur et cognitif d'enfants HC traités de façon précoce. Nos résultats montrent que ce groupe de patients se développe de façon normale, ce qui justifie le dépistage dont il a bénéficié.

Pour évaluer les sujets, nous nous sommes servi des échelles de développement de Griffiths [2] qui constituent la tentative la plus récente d'évaluation du fonctionnement intellectuel d'enfants âgés de un mois à huit ans et qui recouvrent cinq ou six domaines spécifiques représentés de façon égale à chaque niveau d'âge: la locomotion, le développement personnel et social, l'audition et le langage, la coordina- tion oculo-manuelle, le raisonnement non-verbal et le raisonnement pratique (tableau $I)$. Les résultats sont additionnés et fournissent un score global. Ceci permet d'apprécier un large spectre d'habileté ce qui améliore probablement les qualités prédictives du test $[3,4]$. Les 45 premiers patients ont été évalués à douze et dix-huit mois; par la suite ce dernier niveau fut préféré et les enfants ont été successivement examinés à trois et cinq ans. A sept ans nous avons utilisé le Wechsler Intelligence Scale for Children-Revised (WISC-R), instrument choisi lors du I $^{\text {er }}$ Symposium International sur l'HC à Québec en I979 [5] comme une mesure additionnelle acceptée par les différentes équipes engagées dans des programmes prospectifs. Nous prévoyons un suivi à neuf ans et au cours de l'adolescence.

Les normes des échelles de Griffiths étant britanniques, nous avons utilisé des groupes témoins sélectionnés selon les variables suivantes : âge de l'enfant, sexe, rang dans la famille, origine canadienne française, présence de la mère à la maison, double index socioéconomique de Hollingshead (qui tient compte des études réalisées et de la fonction occupée). Nous avons appliqué cette classification au père et à la mère, en utilisant pour celleci le dernier emploi maintenu pendant une période de six.mois.

Nos patients forment un groupe homogène et ont tous 'été traités avant l'âge d'un mois..Leur développement a été régulièrement évalué par une équipe permanente. 
Tableau 1

RÉSULTATS AUX ÉCHELLES DE GRIFFITHS ET AU WISC-R

\begin{tabular}{|c|c|c|c|c|c|c|c|c|c|}
\hline $\begin{array}{l}\text { âge } \\
\text { (ans) }\end{array}$ & $N$ & $\begin{array}{l}\text { loco- } \\
\text { motion }\end{array}$ & social & langage & $\begin{array}{l}\text { coordi- } \\
\text { nation } \\
\text { manuelle }\end{array}$ & $\begin{array}{l}\text { raisonnement } \\
\text { non verbal }\end{array}$ & $\begin{array}{l}\text { raisonnement } \\
\text { pratique }\end{array}$ & $\begin{array}{l}\text { QD global } \\
\text { (moyenne } \\
\pm \text { ETM) }\end{array}$ & $\begin{array}{l}\text { distri- } \\
\text { bution }\end{array}$ \\
\hline 1 & $\begin{array}{l}45 \mathrm{H} \\
37 \mathrm{~T}\end{array}$ & $\begin{array}{l}119 \\
119\end{array}$ & $\begin{array}{l}111 \\
111\end{array}$ & $\begin{array}{l}111 \\
111\end{array}$ & $\begin{array}{l}111 \\
113\end{array}$ & $\begin{array}{l}117 \\
119\end{array}$ & - & $\begin{array}{l}115 \pm 1,5 \\
115 \pm 1,4\end{array}$ & $\begin{array}{r}(88-133) \\
(102-139)\end{array}$ \\
\hline 1,5 & $\begin{array}{l}98 \mathrm{H} \\
41 \mathrm{~T}\end{array}$ & $\begin{array}{l}109 \\
112\end{array}$ & $\begin{array}{l}104^{* *} \\
108=\end{array}$ & $\begin{array}{l}100^{\text {*.*** }} \\
108^{2}\end{array}$ & $\begin{array}{l}106 \\
107\end{array}$ & $\begin{array}{l}105^{* \cdots *} \\
114\end{array}$ & $\begin{array}{l}- \\
- \\
-\end{array}$ & $\begin{array}{l}105 \pm 1,2 \cdots \cdots \\
110 \pm 1,0\end{array}$ & $\begin{array}{l}(64-122) \\
(94-121)\end{array}$ \\
\hline 3 & $\begin{array}{l}69 \mathrm{H} \\
40 \mathrm{~T}\end{array}$ & $\begin{array}{l}102 * * \\
111\end{array}$ & $\begin{array}{l}110^{\circ} \\
118^{\circ}\end{array}$ & $\begin{array}{l}102 * \cdots \\
128\end{array}$ & $\begin{array}{l}99^{*} \\
107\end{array}$ & $\begin{array}{l}105^{* \cdots *} \\
116^{\prime \prime}\end{array}$ & $\begin{array}{l}90 \ldots * \\
103\end{array}$ & $\begin{array}{l}101 \pm 2,3 \cdots \cdots \\
114 \pm 2,2\end{array}$ & $\begin{array}{l}(55-140) \\
(82-139)\end{array}$ \\
\hline 5 & $\begin{array}{l}51 \mathrm{H} \\
45 \mathrm{~T}\end{array}$ & $\begin{array}{l}100 \\
105\end{array}$ & $\begin{array}{l}114 \\
116\end{array}$ & $\begin{array}{l}106 \\
112\end{array}$ & $\begin{array}{l}101 \\
105\end{array}$ & $\begin{array}{l}100^{* *} \\
110\end{array}$ & $\begin{array}{l}85^{\cdots *} \\
93\end{array}$ & $\begin{array}{l}101 \pm 1,8^{*} \\
107 \pm 1,4\end{array}$ & $\begin{array}{l}(60-123) \\
(83-128)\end{array}$ \\
\hline 7 & $36 \underset{\mathrm{T}^{\prime}}{\mathrm{H}}$ & & & $\begin{array}{r}94 \\
100\end{array}$ & & $\begin{array}{l}105 \\
100\end{array}$ & & $\begin{array}{r}99 \pm 2,5 \\
100\end{array}$ & $(74-133)$ \\
\hline
\end{tabular}

"p<0,05, " $p<0,01, " * p<0,005,{ }^{* * *} p<0,001 . H=$ Hypothyroidiens. $T=$ Témoins. $T^{\prime}=$ Norme théorique de 100.

Seuls les HC qui satisfaisaient aux critères de définition du déficit, de respect de la thérapie, de croissance générale satisfaisante et de stabilité du milieu familial ont été conservés dans la présentation de nos données. Lors de l'évaluation à sept ans, nous n'avons pas constitué de groupe témoin, le test de WISC-R possédant des normes nord-américaines largement utilisées dans le milieu. En valeur absolue, les résultats de nos deux groupes d'enfants ont toujours atteint ou dépassé la moyenne de roo estimée normale pour la population (tableau I). A l'âge d'un an, aucune différence n'apparaît entre les résultats moyens du groupe d'HC et des sujets témoins. A partir de dix-huit mois, l'on observe une différence de quelques points en faveur des témoins, ceci se maintient à trois et cinq ans et se retrouve dans la plupart des échelles. Au niveau des quotients globaux, ces différences sont significatives (test de $t, p$ o.or). A l'âge de sept ans, avec le WISC-R les $\mathrm{HC}$ obtiennent un quotient intellectuel (QI) de 99.

Le Test Statistique Hotelling $\mathrm{T}^{2}$ calculé pour les cinq échelles évaluées à l'âge de dix-huit mois différencie nos deux groupes de façon très significative. L'analyse discriminante indique que les domaines qui distinguent les $\mathrm{HC}$ sont le raisonnement non-verbal, l'audition-langage et le développement social (voir tableau I). A l'âge de trois ans, le Hotelling $\mathrm{T}^{2}$ est calculé $m / s n^{\circ}$ 5, vol. 2, mai 86

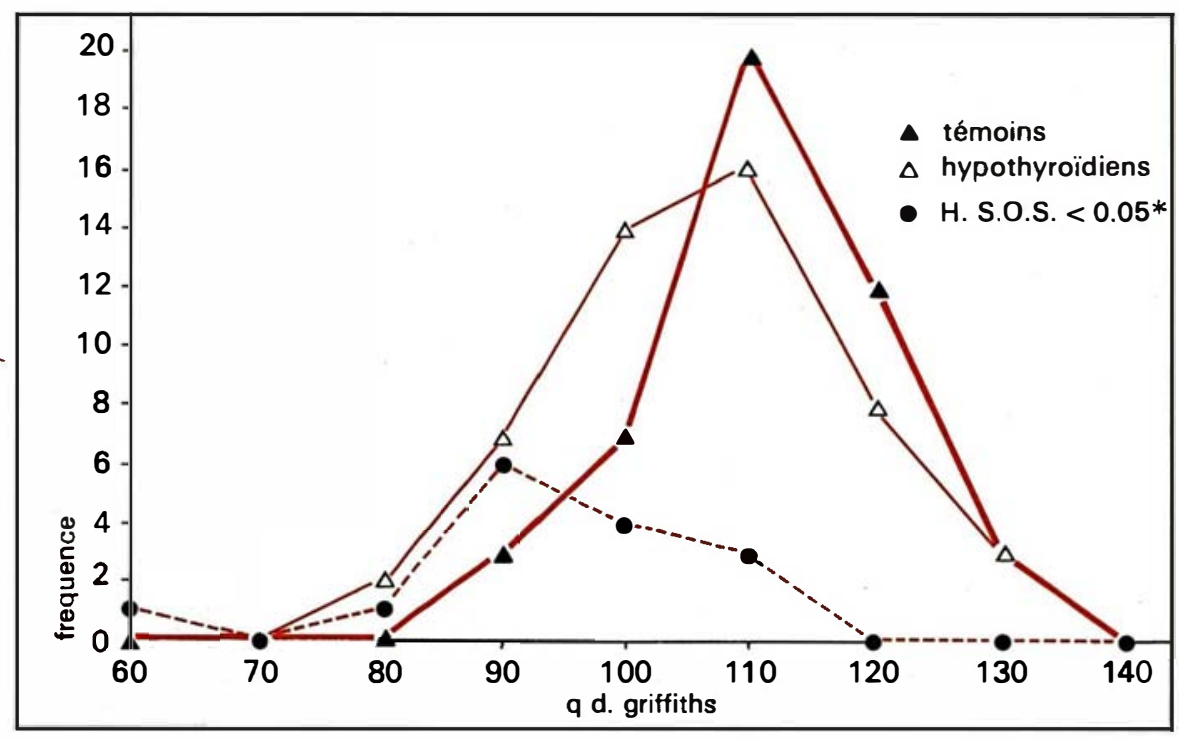

Figure 1. Distribution des quotients de développement à cinq ans.

- Patients dont la valeur de surface osseuse est inférieure à $0,05 \mathrm{~cm}^{2}$.

\begin{tabular}{|c|c|c|c|c|c|c|c|c|}
\hline \multicolumn{9}{|c|}{$\begin{array}{c}\text { Tableau II } \\
\text { QUOTIENT DE DĖVELOPPEMENT ET SURFACE OSSEUSE }\end{array}$} \\
\hline \multirow{3}{*}{$\begin{array}{l}\text { âge } \\
\text { (ans) }\end{array}$} & \multicolumn{6}{|c|}{ surface osseuse } & \multirow{2}{*}{\multicolumn{2}{|c|}{ témoins }} \\
\hline & \multicolumn{3}{|c|}{$\mathrm{HC} \leqq 0,05 \mathrm{~cm}^{2}$} & \multicolumn{3}{|c|}{$\mathrm{HC} \geqq 0,05 \mathrm{~cm}^{2}$} & & \\
\hline & QD & $N$ & distribution & QD & $N$ & distribution & QD & $\mathrm{N}$ \\
\hline $\begin{array}{l}1 \\
1,5 \\
3 \\
5 \\
7\end{array}$ & $\begin{array}{r}107 * * \\
97 \cdots \\
88^{* \cdots *} \\
90 \cdots * \\
88^{* \cdots *}\end{array}$ & $\begin{array}{r}12 \\
22 \\
18 \\
16 \\
8\end{array}$ & $\begin{array}{l}(88-125) \\
(63-110) \\
(54-103) \\
(59-110) \\
(74-98)\end{array}$ & $\begin{array}{l}118 \\
107^{*} \\
106^{*} \\
106 \\
104\end{array}$ & $\begin{array}{l}21 \\
41 \\
33 \\
25 \\
12\end{array}$ & $\begin{array}{r}(105-133) \\
(83-119) \\
(83-140) \\
(92-124) \\
(88-127)\end{array}$ & $\begin{array}{l}115 \\
110 \\
114 \\
107 \\
-\end{array}$ & $\begin{array}{l}37 \\
41 \\
40 \\
45 \\
-\end{array}$ \\
\hline
\end{tabular}

Deux groupes d'HC ont été formés d'après la valeur de surface osseuse : le point de séparation de $0.05 \mathrm{~cm}^{2}$ a été établi en fonction de la distribution cumulée des données mises en logarithmiques. $p<0,05$, " $p<0,01,{ }^{*} " p<0,001$. 
sur six échelles. Tous les résultats sont significativement plus bas chez nos patients. A l'âge de cinq ans, seuls deux domaines différencient les HC des témoins : le raisonnement pratique et le raisonnement non-verbal. A l'âge de sept ans, le test de WISC-R nous fournit deux sous-quotients : le score verbal où les HC atteignent 94 et le nonverbal où ils ont 105 .

Conscients des limites de comparaison patients/témoins, nous avons au cours des années réussi à examiner dix-neuf frères ou sœurs de nos patients au même âge et avec le même test. Les résultats obtenus confirment les données du tableau $I$.

Malgré les bons résultats moyens du groupe d'HC, certains sujets montrent un développement plus lent. Ceci se $r$ eflète dans la distribution des quotients de développement (QD) à toutes nos évaluations [6] et est illustré dans la figure $I$ où nous comparons $5 \mathrm{I} \mathrm{HC}$ et 45 témoins à l'âge de cinq ans. Il est logique de penser qu'une atteinte fœtale plus importante chez certains patients explique ces données.

Le tableau symptomatique spécifique [7] du nourrisson HC n'explique pas le niveau de performance des patients, pas plus que la relative précocité de l'initiation de la thérapie. Les taux de triiodothyronine $\left(\mathrm{T}_{3}\right)$ et de TSH ne sont pas des facteurs prédictifs et il faut atteindre un nombre important de sujets pour obtenir une corrélation significative entre le $\mathrm{T}_{4}$ et les $\mathrm{QD}$.

Par contre, si l'on examine la maturation osseuse des patients [8] au moment du diagnostic, c'est-à-dire à environ un mois de vie et que l'on calcule la surface des épiphyses du genou selon la méthode rapportée par J. Letarte [7], nous mettons en évidence l'existence d'un groupe de sujets de performance intellectuelle significativement plus faible à tous les niveaux d'évaluation que celle des autres patients (tableau II et figure $I$ ). Ces enfants n'ont aucun point d'ossification dans dix-huit cas et de très petits points dans cinq cas. Il nous faut préciser toutefois que trois patients sans point d'ossification obtiennent de bons résultats. Cette mesure nous semble être pour le moment la plus prédictive du niveau de performances intellec- tuelles de jeunes patients.

De nombreux groupes sont maintenant engagés dans des programmes comparables au nôtre mais il est rare de retrouver les conditions dont nous bénéficions: homogénéité d'un groupe suffisamment important et suivi assuré par une même équipe.

Le New-England Collaborative Study a toujours obtenu [9] des QD élevés comparables chez les $\mathrm{HC}$ et les témoins, malgré l'utilisation d'une batterie de tests complexe. Curieusement, aucune corrélation entre les paramètres biologiques et psychologiques n'a été rapportée, et c'est la seule équipe dont les conclusions soient aussi optimistes. Au contraire, Farriaux [1o], Rochiccioli [II] et Rovet [12] obtiennent des résultats similaires aux nôtres. La publication récente des travaux d'Alm [13] nous permet de placer nos données dans une juste perspective : utilisant le même test de Griffiths, il a évalué des enfants identifiés a posteriori comme HC. Ces enfants n'ont donc pas été dépistés à la naissance mais identifiés rétrospectivement en analysant quatre ans plus tard leurs échantillons sanguins obtenus aux fins de dépistage de la phénylcétonurie; I4 enfants, diagnostiqués par le médecin de famille entre l'âge d'un et de quarante et un mois, obtiennent à l'âge de quatre ans et demi un $\mathrm{QD}$ moyen de 87. Cela illustre les progrès accomplis puisque nos 5 I patients, âgés de cinq ans et tous traités avant un mois obtiennent une moyenne de ior.

En conclusion, non seulement le terme de crétinisme a définitivement disparu pour définir l'HC mais l'on constate que l'ensemble des enfants ne présente aucun retard mental. Il faut reconnaître cependant la présence d'un très petit nombre de patients porteurs d'un déficit plus marqué au moment du diagnostic.

Il serait présomptueux de prédire avec exactitude l'évolution de nos sujets mais les résultats actuels sont prometteurs : le développement général des hypothyroïdiens congénitaux traités précocement se poursuit de façon régulière. L'intégration scolaire de nos premiers patients est tout à fait normale

\section{REFERENCES}

I. Dussault JH. Hypothyroïdie congénitale : une éradication complète est-elle possible ? médecine/sciences $1985 ; 1: 261-5$.

2. Griffiths R. The Abilities of Young Children: London Child Development Research Center. London: Young and Sons, 1970.

3. Hindley CB, Owen CF. The extent of individual changes in IQ for ages between 6 months and 17 years, in a British Longitudinal Sample. 7 Child Psychol Psychiatr 1978; 19 : 329-50.

4. McCall RB. The development of intellectual functioning in infancy and the prediction of later IQ. In: Osof sky JD, eds. Handbook of Infant Development. New York: Wiley \& Sons, 1979: 707-4I.

5. Burrow GH, Dussault JH. Neonatal thyroid screening. New York: Raven Press, 1980: 309

6. Glorieux J, La Vecchio FA. Psychological and neurological development in congenital hypothyroidism. In: Dussault JH, Walker P, eds. Congenital Hypothyroidism. New York: Marcel Dekker, 1983: 4II-30.

7. Letarte J, Lafranchi S. Clinical features of congenital hypothyroidism. In: Dussault JH Walker P, eds. Congenital Hypothyroidism, New York: Marcel Dekker, 1983: $35^{1-83}$.

8. Wolter R, Noël P, De Cock P, et al. Neuropsychological study in treated thyroid dysgenesis. Acta Paediatr Scand 1980; suppl 277: 41.

9. New England congenital hypothyroidism: collaborative characteristics of infantile hypothyroidism discovered on neonatal screening. 7 Pediatr 1984; 104: 539-44.

Io. Farriaux JP. Communication personnelle.

II. Rochiccioli P, Roge B, Alexandre M, et al. Résultats du développement psychomoteur des hypothyroïdies dépistées à la naissance. Arch Fr Pédiatr 1983; $40: 537-41$.

12. Rovet JF, Westbrook DL, McErlich RM. Neonatal thyroid deficiency: Early temperamental and cognitive characteristics. 7 Am Acad Child Psychiatry 1984; I: 10-22.

I3. Alm J, Hagenfeldt L, Larsson 'A, Lundberg K. Incidence of congenital hypothyroidism: Retrospective study of neonatal laboratory screening versus clinical symptons as indicators leading to diagnosis. $\mathrm{Br}$ Med 7 1984; 289 : I 17 I-5

\section{TIRES A PART}

J. Glorieux : Centre de Recherche Pédiatrique, Hôpital Sainte-Justine, 3175, Chemin CôteSainte-Catherine, Montréal, Québec, Canada, $\mathrm{H}_{3} \mathrm{~T}_{1} \mathrm{C}_{5}$. 